\title{
Repelência de produtos fitossanitários usados na cana-de-açúcar e seus efeitos na emergência de Trichogramma galloi ${ }^{1}$
}

\author{
Repellence of pesticides used in sugarcane and their effects on the emergence of \\ Trichogramma galloi
}

\author{
Marina de Rezende Antigo ${ }^{2}$, Harley Nonato de Oliveira $^{3 *}$, Geraldo Andrade Carvalho ${ }^{4}$ e Fabricio Fagundes \\ Pereira $^{2}$
}

\begin{abstract}
RESUMO - Trichogramma galloi Zucchi é um importante parasitoide de Diatraea saccharalis (Fabricius), principal praga da cana-de-açúcar. Como vários produtos químicos são utilizados no manejo dessa cultura, o presente trabalho objetivou avaliar a repelência de alguns produtos fitossanitários sobre adultos do parasitoide $T$. galloi e seu efeito na emergência dos descendentes. Os ensaios foram conduzidos em câmara climática a $23 \pm 2^{\circ} \mathrm{C}$, UR de $60 \pm 10 \%$ e fotofase de $14 \mathrm{~h}$. Os produtos avaliados foram: inseticidas clorantraniliprole, etiprole, tiametoxam, triflumurom, lambda-cialotrina+tiametoxam, fipronil e Metarhizium anisopliae, herbicidas clomazone e diurom+hexazinona e os reguladores de crescimento de plantas sulfometurom-metílico e trinexapaque-etílico, nas maiores concentrações recomendadas à cana-de-açúcar. Para a avaliação de repelência de cada produto, realizou-se teste com livre chance de escolha, onde 30 ovos de $D$. saccharalis foram imersos em água destilada e outros 30 na calda do produto a ser testado. Cada uma das massas de ovos destes dois tratamentos foi transferida para um tubo de vidro, sendo os mesmos interligados por um conector de papel com um orifício central, por onde um tubo menor foi acoplado para liberação de três fêmeas de T. galloi, previamente alimentadas. O delineamento experimental foi inteiramente casualizado, com quinze repetições para cada produto avaliado. Avaliou-se a repelência, o número médio de ovos parasitados por fêmea e a emergência. Clorantraniliprole, triflumurom e sulfometurom-metílico não repeliram o parasitoide e nem afetaram o número de ovos parasitados. Para a emergência, verificou-se que os produtos M. anisopliae, tiametoxam, clomazone, diurom+hexazinona e sulfometurom-metílico não afetaram essa característica biológica.
\end{abstract}

Palavras-chave: Cana-de-açúcar. Pragas. Controle biológico.

\begin{abstract}
Trichogramma galloi Zucchi is an important parasitoid of Diatraea saccharalis (Fabricius), a major pest of sugarcane. As various chemical products are used in the management of this crop, this study aimed to evaluate the repellence of some pesticides on adults of the parasitoid T. galloi, and its effect on the emergence of their offspring. The tests were carried out in a climatic chamber at $23 \pm 2{ }^{\circ} \mathrm{C}$, with a relative humidity of $60 \pm 10 \%$ and a photo period of 14 hours. The products evaluated were: insecticides clorantraniliprole, ethiprole, thiamethoxam, triflumuron, lambda-cyhalothrin+thiamethoxam, fipronil and Metarhizium anisopliae; the herbicides clomazone and diuron+hexazinone; and the plant-growth regulators methyl-sulfometuron, and ethyl-trinexapac, at the highest concentrations recommended for sugarcane. To evaluate the repellence of each product, a free-choice test was carried out with, where 30 eggs of $D$. saccharalis were immersed in distilled water and another 30 in a mixture of the product to be tested. Each mass of eggs from the two treatments was transferred to a glass tube, these being connected by a paper connector with a central hole through which a smaller tube was attached to allow the release of three females of T. galloi which had previously been fed. The experimental design was completely randomized, with fifteen repetitions for each product evaluated. Repellence, average number of parasitised eggs per female and emergence rates were all evaluated. Clorantraniliprole, triflumuron and methyl-sulfometuron did not repel the parasitoid nor affect the number of parasitized eggs. As for emergence, it was found that M. anisopliae, thiamethoxam, clomazone, diuron+hexazinone and methyl-sulfometuron did not affect this biological characteristic.
\end{abstract}

Key words: Sugarcane. Pest. Biological control.

\footnotetext{
*Autor para correspondência

${ }^{1}$ Recebido para publicação em 09/04/2012; aprovado em 16/04/2013

Parte da Dissertação de Mestrado do primeiro autor no Curso de Pós-Graduação em Entomologia e Conservação da Biodiversidade na UFGD, bolsa financiada pela CAPES e pesquisa financiada pelo CNPq e Embrapa

${ }^{2}$ Universidade Federal da Grande Dourados/UFGD, Brasil, mahrezende@hotmail.com, fabriciofagundes@ufgd.edu.br

${ }^{3}$ Embrapa Agropecuária Oeste, Brasil, harley.oliveira@embrapa.br

${ }^{4}$ Universidade Federal de Lavras/UFLA, Câmpus Universitário, Caixa Postal 3037, Lavras-MG, Brasil, 37.200-000, gacarval@ den.ufla.br
} 


\section{INTRODUÇÃO}

A cultura da cana-de-açúcar (Saccharum officinarum) (Linnaeu) é de grande importância econômica no Brasil, atingindo aproximadamente 10 milhões de hectares cultivados com cerca de 700 milhões de toneladas de produção (INSTITUTO BRASILEIRO DE GEOGRAFIA E ESTATÍSTICA, 2011).

A broca-da-cana-de-açúcar, Diatraea saccharalis (Fabricius) (Lepidoptera: Crambidae), é a praga mais importante dessa cultura, sendo a fase larval a que causa prejuízos (COSTA; FRANCEZ; SÁ, 2010). As lagartas de primeiro instar alimentam-se inicialmente das folhas do cartucho, raspando-as, e migrando posteriormente em direção ao colmo. As lagartas penetram no colmo na região dos nós, próximo às gemas e abrem galerias ascendentes na região do palmito, com orifícios verticais e transversais (PINTO; CANU; SANTOS, 2006). Esse comportamento dificulta seu manejo, pois o inseto fica protegido contra as pulverizações de inseticidas.

O controle biológico de $D$. saccharalis é extremamente importante, especialmente quando se emprega o endoparasitoide larval Cotesia flavipes (Cameron) (Hymenoptera: Braconidae) (BROGLIOMICHELETTI et al., 2007). No entanto, Pinto et al. (2003) relataram que o controle utilizando o parasitoide de ovos Trichogramma, tem como grande vantagem o fato desse inimigo natural poder reduzir a população da praga antes que algum dano seja causado à cultura. $\mathrm{O}$ parasitoide de ovos Trichogramma galloi Zucchi (Hymenoptera: Trichogrammatidae) constitui uma importante alternativa de controle biológico (BOTELHO et al., 1999), pois este inimigo natural ocorre naturalmente no Brasil e chega a causar parasitismo em ovos de D. saccharalis superior a 90\% (PINTO et al., 2006). Na literatura há trabalhos evidenciando a eficiência de $T$. galloi no controle de ovos de $D$. saccharalis em locais com altas infestações da broca, e, sobretudo, a ação sinérgica por ocasião da sua associação com C. flavipes (BOTELHO et al., 1999). Além da eficiência no controle em condições de campo, T. galloi pode ser facilmente criado em laboratório, o que potencializa o uso desse inimigo natural.

Uma série de produtos fitossanitários, como os inseticidas, herbicidas e os reguladores de crescimento de plantas são utilizados na cultura da cana e estes podem afetar os inimigos naturais, comprometendo assim a eficiência no controle das pragas. Os produtos químicos devem ser associados a outras táticas de controle quando apresentarem algum grau de seletividade, o que é a chave para o manejo integrado de pragas (SANTOS; BUENO; BUENO, 2006).

Além de efeitos fisiológicos, os inseticidas e outros produtos químicos podem afetar características biológicas de predadores e parasitoides, tais como a fecundidade, a longevidade, a taxa de desenvolvimento e a razão sexual. Efeitos negativos no comportamento também são vistos, principalmente na capacidade de busca e mobilidade, além de ocorrerem modificações no comportamento reprodutivo e alimentar de inimigos naturais (FOERSTER, 2002).

Este estudo objetivou verificar se produtos fitossanitários usados na cultura da cana podem afetar T. galloi, repelindo ou mesmo influenciando seu parasitismo e sua emergência em ovos de $D$. saccharalis, com o intuito de gerar informações que possam auxiliar na atuação e/ou manutenção desse organismo benéfico nos agroecossistemas por meio da aplicação de produtos seletivos.

\section{MATERIAL E MÉTODOS}

Para a realização dos bioensaios foram escolhidos os seguintes produtos fitossanitários utilizados na cultura da cana-de-açúcar: os inseticidas clorantraniliprole $(350 \mathrm{~g} / \mathrm{kg}$ - WG) ( ${ }^{\circ}$ registro MAPA 8909), etiprole (200 g/L - SC) $\left(\mathrm{n}^{\mathrm{o}}\right.$ registro MAPA 10806), tiametoxam $(250 \mathrm{~g} / \mathrm{kg}-\mathrm{WG})$ $\left(\mathrm{n}^{\mathrm{o}}\right.$ registro MAPA 10098), triflumurom (480 $\left.\mathrm{g} / \mathrm{L}-\mathrm{SC}\right)$ $\left(\mathrm{n}^{\mathrm{o}}\right.$ registro MAPA 4899), lambda-cialotrina+tiametoxam $(106 \mathrm{~g} / \mathrm{L}+141 \mathrm{~g} / \mathrm{L}-\mathrm{SC})\left(\mathrm{n}^{\circ}\right.$ registro MAPA 6105), fipronil $(800 \mathrm{~g} / \mathrm{kg}-\mathrm{WG})\left(\mathrm{n}^{\circ}\right.$ registro MAPA 5794) e Metharizium anisopliae (50 g/kg - WP) ( $\mathrm{n}^{\circ}$ registro MAPA 6605), os herbicidas clomazone $(800 \mathrm{~g} / \mathrm{L}-\mathrm{EC})\left(\mathrm{n}^{\circ}\right.$ registro MAPA 8008) e diurom+hexazinona $(488 \mathrm{~g} / \mathrm{kg}+142 \mathrm{~g} / \mathrm{kg}-\mathrm{WP})\left(\mathrm{n}^{\circ}\right.$ registro MAPA 958388) e os reguladores de crescimento de plantas sulfometurom-metílico $(750 \mathrm{~g} / \mathrm{kg}-\mathrm{WG})\left(\mathrm{n}^{\mathrm{o}}\right.$ registro MAPA 8198$)$ e trinexapaque-etílico $(250 \mathrm{~g} / \mathrm{L}$ EC) (nº registro MAPA 296). O número entre parênteses refere-se à concentração do ingrediente ativo no produto comercial, sendo que todas as soluções avaliadas estavam nas concentrações máximas indicadas para a cultura, conforme metodologia recomendada pela "International Organization for Biological Control and Integrated Control of Noxious Animals and Plants" (STERK et al., 1999).

Os testes com livre chance de escolha basearamse nas metodologias propostas por Gonçalves-Gervásio e Vendramim (2004) e Potrich et al. (2009) com algumas modificações, descritas a seguir. Uma repetição foi composta por 60 ovos de D. saccharalis criada em dieta artificial. Esses foram ovipositados em folhas de papel jornal, sendo que 30 destes ovos foram imersos em água destilada e os outros 30 na calda química de cada produto testado durante cinco segundos. Após secarem, as massas de ovos tratadas com produtos ou água destilada foram transferidas para tubos de vidro $(8,5 \mathrm{~cm}$ de altura $\times 2,5 \mathrm{~cm}$ de diâmetro) distintos, os quais foram interligados um ao outro por um conector de papel contendo um orifício para 
inserção de um tubo com dimensões menores $(4,0 \mathrm{~cm}$ de altura $\mathrm{x} 0,7 \mathrm{~cm}$ de diâmetro). Os tubos menores possuíam três fêmeas de T. galloi previamente alimentadas, com idade de $24 \mathrm{~h}$. O parasitismo foi permitido por $24 \mathrm{~h}$, sendo que após esse período as fêmeas foram retiradas dos tubos e os mesmo foram fechados com filme de PVC. Uma unidade experimental, contendo somente ovos imersos em água destilada (controle) também foi montada, buscando avaliar o potencial de parasitismo e emergência de $T$. galloi.

Os bioensaios foram realizados em câmara climática a $23 \pm 2{ }^{\circ} \mathrm{C}$, UR de $60 \pm 10 \%$ e fotofase de $14 \mathrm{~h}$. Para garantir a entrada de gases ou saída de vapores tóxicos do interior do tubo, orifícios foram feitos nos rolos de papel, utilizando-se para isso alfinetes entomológicos. $\mathrm{O}$ delineamento experimental foi o inteiramente casualizado, com quinze repetições para cada produto avaliado. Foi avaliada a porcentagem de parasitismo, o número médio de ovos parasitados por fêmea e a emergência.

O efeito de repelência foi considerado quando no controle era encontrada uma porcentagem de parasitismo significativamente maior que nos ovos tratados com o produto. Os dados referentes ao parasitismo, número médio de ovos parasitados por fêmea e emergência do parasitoide foram submetidos à análise de variância (ANOVA) e as médias dos tratamentos comparadas pelo teste de Tukey ao nível de 5\% de significância, utilizando o programa estatístico ASSISTAT, beta 7.6, desenvolvido pela Universidade Federal de Campina Grande, PB (ASSISTAT, 2011).

\section{RESULTADOS E DISCUSSÃO}

\section{Parasitismo}

Para os inseticidas clorantraniliprole, triflumurom, fipronil e o regulador de crescimento sulfometurommetilico, não foi verificado efeito significativo de repelência em relação ao parasitismo de T. galloi, uma vez que não houve diferença no número de ovos parasitados entre os ovos tratados com esses produtos e aqueles imersos em água (Tabela 1). Dentre os tratamentos, fipronil provocou a morte rápida das fêmeas e em consequência houve a redução no número médio de ovos parasitados (Tabela 1). Davies, Pufke e Zalucki (2009) também verificaram que em plantação de algodoeiro o índice de parasitismo de Trichogramma spp. em ovos de Helicoverpa spp., declinou de forma acentuada após a aplicação deste inseticida.

Os ovos tratados com clorantraniliprole apresentaram parasitismo similar aos da testemunha (Tabela 1), uma vez que a porcentagem de parasitismo observada nos ovos tratados com este inseticida não diferiu estatisticamente daqueles imersos apenas em água destilada. Este produto afetou o parasitismo de Trichogramma pretiosum Riley (Hymenoptera: Trichogrammatidae) em ovos de Anagasta kuehniella (Zeller) (Lepidoptera: Pyralidae), pois na dosagem de 85,7 g p.c. ha ${ }^{-1}$ a redução variou de 30 a $79 \%$, provavelmente devido aos ingredientes inertes contidos na formulação de clorantraniliprole (GRUTZMACHER et al., 2011).

Em relação à triflumurom, semelhante aos resultados encontrados neste trabalho (Tabela 1), Carvalho et al. (2010) verificaram que este produto não reduziu o número de ovos de A. kuehniella parasitados por $T$. pretiosum quando ofertados 24 e 48 horas após serem tratados com esse inseticida. Da mesma forma, Maia et al. (2010) classificaram triflumurom como inócuo em testes de seletividade para Trichogramma atopovirilia Oatman \& Platner (Hymenoptera: Trichogrammatidae) em ovos de A. kuehniella. A inocuidade desse inseticida ao parasitismo de Trichogramma spp., talvez possa estar relacionada ao seu modo de ação, pois sendo um composto pertencente ao grupo químico das benzoiluréias, ele inibe a síntese de quitina em insetos imaturos, não afetando, portanto, os adultos.

Fipronil é um inseticida pertencente ao grupo químico fenilpirazol, um antagonista do neurotransmissor GABA (ácido gama-aminobutírico), atuando através da supressão da permeabilidade da membrana da célula nervosa ao íon $\mathrm{Cl}^{-1}$, gerando uma hiperexcitação do sistema nervoso central dos insetos (GALLO et al., 2002), que leva o inseto rapidamente à morte. Como fipronil não repeliu o parasitoide, os mesmos conseguiram entrar em contato com os ovos tratados e sendo rápido o modo de ação deste produto, as fêmeas do parasitoide ovipositaram pouco antes de morrer, sendo verificada uma redução no número médio de ovos parasitados por fêmea. Davies, Pufke e Zalucki (2009) também verificaram que em plantação de algodão o índice de parasitismo observado de Trichogramma spp. em ovos de Helicoverpa spp., declinou de forma acentuada após a aplicação deste inseticida.

O tratamento com o regulador de crescimento sulfometurom-metílico não provocou a redução no número de ovos parasitados (Tabela 1).

$\mathrm{O}$ efeito de repelência ocorreu com a aplicação dos seguintes tratamentos: M. anisopliae, tiametoxam, lambda-cialotrina+tiametoxam, etiprole, os herbicidas clomazone e diurom+hexazinona e o regulador de crescimento trinexapaque-etílico, pois deferiram estatisticamente dos demais, resultando em menor \% de parasitismo (Tabela 1). Dentre eles, tiametoxam, lambda-cialotrina+tiametoxam, etiprole e clomazone proporcionaram uma redução acentuada no número médio de ovos parasitados (Tabela 1), o que pode ser explicado pela rápida mortalidade dos parasitoides que entraram em contato com estes compostos. 
Tabela 1 - Parasitismo, número de ovos parasitados e emergência de Trichogramma galloi (Hymenoptera: Trichogrammatidae) em ovos de Diatraea saccharalis (Lepidoptera: Crambidae) previamente tratados com produtos fitossanitários usados em cana-de-açúcar ou água destilada como controle, em teste de livre chance de escolha

\begin{tabular}{|c|c|c|c|c|c|}
\hline \multirow{2}{*}{ Produtos } & \multicolumn{2}{|c|}{ Parasitismo (\%) } & \multirow{2}{*}{$\mathrm{N}^{\mathrm{o}}$ médio de ovos parasitados/fêmea } & \multicolumn{2}{|c|}{ Emergência (\%) } \\
\hline & $\mathrm{OIA}^{1}$ & $\mathrm{OIP}^{2}$ & & $\mathrm{OIA}^{1}$ & $\mathrm{OIP}^{2}$ \\
\hline \multicolumn{6}{|c|}{ INSETICIDAS } \\
\hline Clorantraniliprole & $21,78 \mathrm{a}$ & $23,78 \mathrm{a}$ & $6,83 \mathrm{AB}$ & 79,93 a & $54,10 \mathrm{~b}$ \\
\hline Metarhizium anisopliae & $27,78 \mathrm{a}$ & $17,10 \mathrm{~b}$ & $6,73 \mathrm{AB}$ & $70,10 \mathrm{a}$ & $53,97 \mathrm{a}$ \\
\hline Triflumurom & $17,78 \mathrm{a}$ & $18,00 \mathrm{a}$ & $5,37 \mathrm{AB}$ & $56,89 \mathrm{a}$ & $0,00 \mathrm{~b}$ \\
\hline Fipronil & $5,78 \mathrm{a}$ & $6,89 \mathrm{a}$ & $1,90 \mathrm{~B}$ & $64,01 \mathrm{a}$ & $0,00 \mathrm{~b}$ \\
\hline Tiametoxam & $14,00 \mathrm{a}$ & $3,33 \mathrm{~b}$ & $2,60 \mathrm{~B}$ & $67,58 \mathrm{a}$ & $65,08 \mathrm{a}$ \\
\hline Lambda-cialotrina+Tiametoxam & $10,00 \mathrm{a}$ & $0,00 \mathrm{~b}$ & $1,50 \mathrm{~B}$ & 74,11 & - \\
\hline Etiprole & $14,67 \mathrm{a}$ & $8,44 \mathrm{~b}$ & $3,47 \mathrm{~B}$ & $69,74 \mathrm{a}$ & $35,52 \mathrm{~b}$ \\
\hline \multicolumn{6}{|c|}{ HERBICIDAS } \\
\hline Clomazone & $11,33 \mathrm{a}$ & $0,89 \mathrm{~b}$ & $1,83 \mathrm{~B}$ & $63,11 \mathrm{a}$ & $50,00 \mathrm{a}$ \\
\hline Diurom+Hexazinona & $39,33 \mathrm{a}$ & $12,67 \mathrm{~b}$ & $7,80 \mathrm{AB}$ & $75,02 \mathrm{a}$ & $49,39 \mathrm{a}$ \\
\hline \multicolumn{6}{|c|}{ REGULADORES DE CRESCIMENTO } \\
\hline Trinexapaque-etílico & $18,89 \mathrm{a}$ & $8,22 \mathrm{~b}$ & $4,07 \mathrm{AB}$ & $54,53 \mathrm{a}$ & $0,00 \mathrm{~b}$ \\
\hline Sulfometurom-metílico & $14,89 \mathrm{a}$ & 19,11 a & $5,10 \mathrm{AB}$ & $45,84 \mathrm{a}$ & $60,98 \mathrm{a}$ \\
\hline Água destilada & $24,67 \mathrm{a}$ & $32,44 \mathrm{a}$ & $8,57 \mathrm{~A}$ & $63,96 \mathrm{a}$ & $53,83 \mathrm{a}$ \\
\hline
\end{tabular}

Médias na mesma linha, seguidas de mesma letra minúscula não diferem entre si e as médias na coluna seguidas de mesma letra maiúscula não diferem entre si pelo teste de Tukey a 5\%; ${ }^{1}$ OIA: ovos imersos em água (controle). ${ }^{2} \mathrm{OIP}$ : ovos imersos no produto

Semelhante ao encontrado neste trabalho, Silva et al. (2010) verificaram repelência no parasitismo de $T$. pretiosum em ovos de A. kuehniella quando estes foram tratados com M. anisopliae. Da mesma forma, BroglioMicheletti, Santos, Pereira-Barros (2006) observaram redução no parasitismo de T. galloi em ovos de D. saccharalis tratados com esse produto. Esses autores ainda ressaltaram que a presença do fungo pode dificultar o reconhecimento dos ovos do hospedeiro pelo parasitoide, o que explicaria a menor porcentagem de parasitismo observada nos ovos tratados nesse trabalho. Diferentemente dos resultados obtidos neste estudo, foi observado que o número de ovos de A. kuehniela parasitados por T. pretiosum não foi afetado pela aplicação de $M$. anisopliae, tampouco foi constatado a ação de repelência (POTRICH et al., 2009).

Referente ao tiametoxam, diferentemente do observado nesse trabalho (Tabela 1), Pratissoli et al. (2009) não verificaram efeito no parasitismo de $T$. pretiosum em ovos de A. kuehniella, Sitotroga cerealella (Oliver) (Lepidoptera: Gelechiidae) e Anticarsia gemmatalis Hübner (Lepidoptera: Noctuidae) tratados com esse produto. Essas diferenças observadas podem estar relacionadas com a estrutura dos ovos dos diferentes hospedeiros (CÔNSOLI; KITAJIMA; PARRA, 1999), pois os ovos de D. saccharalis possuem córion composto de pequenas estruturas irregulares, dispostas assimetricamente, resultando em uma textura áspera com pequenas fraturas na superfície, que pode influenciar no grau de impregnação dos produtos. Outro fator que poderia ter influenciado na divergência dos resultados seria a dosagem de tiametoxam utilizada, uma vez que a de Pratissoli et al. (2009) foi baseada na cultura do tomateiro, que foi menor que a utilizada nessa pesquisa.

Lambda-cialotrina+tiametoxam foi o único tratamento em que não foi constatado parasitismo. $\mathrm{O}$ primeiro composto é do grupo dos piretroides, o qual ligase aos canais de $\mathrm{Na}^{+}$na membrana axônica, retardando o seu fechamento após a formação do impulso nervoso. Com isso, potenciais de ação repetitivos são desencadeados e os insetos morrem devido à hiperexcitabilidade (GALLO et al., 2002). Aliado a isso, piretroides têm sido reportados como repelentes a inimigos naturais (GOULART et al., 2008). Já o neocotinoide tiametoxam, um agonista de acetilcolina do grupo químico dos neonicotinoides, se liga aos receptores nicotínicos da acetilcolina localizados nos neurônios pós-sinápticos e não se degrada imediatamente, como ocorre com a acetilcolina. Portanto, os impulsos nervosos são transmitidos continuamente, levando à hiperexcitação do sistema nervoso e consequentemente 
à morte do inseto (GALLO et al., 2002). Como os dois ingredientes ativos desse inseticida atuam no sistema nervoso do inseto, a integração destes compostos pode ter potencializado seu modo de ação, matando rapidamente os parasitoides que entraram em contato com os ovos tratados ou mesmo pela presença de substâncias voláteis; refletindo assim numa diminuição significativa no número de ovos parasitados, com ausência de emergência (Tabela 1).

Stefanello Júnior et al. (2008) também verificaram redução significativa no número de ovos parasitados por T. pretiosum em ovos de A. kuehniella quando tratados com produto à base de lambda-cialotrina, classificandoo como prejudicial (redução > 99\%).

Notratamentocometiprole, pertencente aomesmo grupo químico de fipronil, foi observada a redução no número de ovos parasitados por fêmeas de $T$. galloi como também a ação de repelência. Provavelmente, estas ações foram decorrentes da maior concentração do ingrediente ativo que fipronil. Verificou-se que clomazone repeliu o parasitoide e também reduziu o número de ovos parasitados. Da mesma forma, Bastos, Almeida e Suinaga (2006) constataram que esse produto também afetou negativamente o parasitismo de T. pretiosum em ovos de S. cerealella e A. kuehniella.

Para o herbicida diurom+hexazinona foi verificado repelência, uma vez que o parasitismo dos ovos da testemunha foi maior que nos ovos tratados com este produto, entretanto, não houve redução no número médio de ovos parasitados por fêmea; para o regulador de crescimento trinexapaque-etílico verificou-se redução na porcentagem de parasitismo, em relação ao controle, caracterizando dessa forma a repelência deste produto a T. galloi. Entretanto, não foram encontrados trabalhos na literatura sobre os efeitos desses produtos em insetos.

\section{Emergência}

Em relação à emergência, não foi verificado efeito dos tratamentos M. anisopliae, tiametoxam, clomazone e diurom+hexazinona sulfometurom-metílico, já que não foram observadas diferenças significativas entre os ovos tratados e o controle (Tabela 1). Resultados semelhantes aos obtidos neste estudo com o fungo $M$. anisopliae foram observados para T. galloi em ovos de D. saccharalis (BROGLIO-MICHELETTI; SANTOS; PEREIRA-BARROS, 2006) e T. atopovirilia em ovos de Spodoptera frugiperda (Smith) (Lepidoptera: Noctuidae) (POLANCZYK et al., 2010) e para $T$. pretiosum em ovos de A. kuehniella, S. cerealella e A. gemmatalis (PRATISSOLI et al., 2009). Em relação à clomazone foi verificado que esse produto também não afetou a emergência de $T$. pretiosum em ovos de $A$. kuehniella (CARMO et al., 2010).
Os produtos que apresentaram reduções sobre a emergênciadeT. galloiforamos tratamentos: clorantraniliprole, triflumurom, fipronil, etiprole e trinexapaque-etílico, sendoque dos ovos tratados com triflumurom, fipronil e trinexapaqueetílico não houve emergência (Tabela 1).

Esse efeito sobre a emergência de $T$. galloi dos ovos tratados com clorantraniliprole poderia ser resultado do seu modo de ação, pois segundo Brugger et al. (2010) esse inseticida atua sobre a musculatura, causa paralisia e finalmente a morte do inseto. No entanto, isso pode ser variável em função da espécie de Trichogramma utilizada e da dosagem, pois Brugger et al. (2010) relataram que esse produto não afetou a emergência de Trichogramma chilonis Ishii (Hymenoptera: Trichogrammatidae) de ovos de Corcyra cephalonica (Stainton) (Lepidoptera: Pyralidae) quando tratados com clorantraniliprole (formulação 20SC em menores concentrações: 80 e $100 \mathrm{mg} \mathrm{L}^{-1}$ ).

Goulart et al. (2008) verificaram que para $T$. pretiosum e T. exiguum em ovos de diferentes hospedeiros tratados com mesmo produto comercial à base de triflumurom, a emergência dos parasitoides não foi diminuída, diferente do observado neste trabalho. No entanto, a dosagem utilizada por este autor (dosagem recomendada para cultura do tomateiro) foi inferior à utilizada neste trabalho.

Foi observado que a emergência de adultos de Cotesia plutellae (Kurdjumov) (Hymenoptera: Braconidae) não foi reduzida quando as pupas foram tratadas com fipronil. Possivelmente, este fato pode estar relacionado à fase do inseto e a proteção pelo casulo (SHI et al., 2004). Os tratamentos, etiprole e trinexapaque-etílico afetaram negativamente a emergência de $T$. galloi.

O efeito de lambda-cialotrina+tiametoxam na emergência de T. galloi não pôde ser determinado devido ao fato dos ovos tratados com esse produto não terem apresentado parasitismo.

\section{CONCLUSÕES}

Nas condições em que este estudo foi realizado, pode-se concluir que:

1. Os tratamentos clorantraniliprole, triflumurom e sulfometurom-metílico foram seletivos, pois não afetaram o parasitismo de $T$. galloi em ovos de $D$. saccharalis, podendo ser utilizados de forma associada em plantios de cana-de-açúcar;

2. Os tratamentos com Metarhizium anisopliae, diurom+hexazinona e trinexapaque-etílico mostraram um efeito negativo no parasitismo, evidenciando a repelência de $T$. galloi em relação a esses produtos; 
3. Os tratamentos fipronil, tiametoxam, lambdacialotrina+tiametoxam, etiprole e clomazone provocaram redução na ação de T. galloi no controle da broca-da-cana;

4. Novos estudos devem ser realizados para que seja confirmado o efeito dos tratamentos em condições de semicampo e campo.

\section{AGRADECIMENTOS}

Á CAPES, ao CNPq e à Embrapa pelo auxílio concedido.

\section{REFERÊNCIAS}

ASSISTAT. Versão beta 7.6. Campina Grande: Universidade Federal de Campina Grande, 2011. Disponível em: < http:// www.assistat.com/indformp.html >. Acesso em: 07 set. 2011.

BASTOS, C. S.; ALMEIDA, R. P.; SUINAGA, F. A. Selectivity of pesticides used on cotton (Gossypium hirsutum) to Trichogramma pretiosum reared on two laboratory-reared hosts. Pest Management Science, v. 62, n. 1, p. 91-98, 2006.

BOTELHO, P. S. M. et al. Associação do parasitóide de ovos Trichogramma galloi Zucchi (Hymenoptera: Trichogrammatidae) e do parasitóide larval Cotesia flavipes (Cam.) (Hymenoptera: Braconidae) no controle de Diatraea saccharalis, (Fabr.) (Lepidoptera: Crambidae) em cana-deaçúcar. Anais da Sociedade Entomológica do Brasil, v. 28, n. 3, p. 491-496, 1999.

BROGLIO-MICHELETTI, S. M. F. et al. Efeito do número de adultos de Trichogramma galloi (Zucchi, 1988) (Hymenoptera: Trichogrammatidae) liberados em semanas sucessivas, para o controle de Diatraea saccharalis (Fabricius, 1794) (Lepidoptera: Crambidae). Ciência e Agrotecnologia, v. 31, n. 1, p. 53-58, 2007.

BROGLIO-MICHELETTI, S. M. F.; SANTOS, A. D. J. N.; PEREIRA-BARROS, J. L. Ação de alguns produtos fitossanitários para adultos de Trichogramma galloi Zucchi, 1988 (Hyenoptera: Trichogrammatidae). Ciência e Agrotecnologia, v. 30, n. 6, p. 1051-1055, 2006.

BRUGGER, K. E. et al. Selectivity of chlorantraniliprole to parasitoid wasps. Pest Management Science, v. 66, n. 10, p. 1075-1081, 2010.

CARMO, E. L. et al. Seletividade de produtos fitossanitários utilizados na cultura da soja para pupas de Trichogramma pretiosum Riley, 1879 (Hymenoptera: Trichogrammatidae). Arquivos do Instituto Biológico, v. 77, n. 2, p. 283-290, 2010.

CARVALHO, G. A. et al. Selectivity of growth regulators and neonicotinoids for adults of Trichogramma pretiosum (Hymenoptera: Trichogrammatidae). Revista Colombiana de Entomologia, v. 36, n. 2, p. 195-201, 2010.
CÔNSOLI, F. L.; KITAJIMA, E. W.; PARRA, J. R. P. Ultrastructure of the natural and factitious host eggs of Trichogramma galloi Zucchi and Trichogramma pretiosum Riley (Hymenoptera: Trichogrammatidae). International Journal of Insect Morphology and Embryology, v. 28, n. 04, p. 211-229, 1999.

COSTA, D. M.; FRANCEZ, A. C. C.; SÁ, R. O. Biologia da broca da cana-de-açúcar (Diatraea saccharalis) (Lepidoptera: Crambidae) em dieta artificial. Ciência et Praxis, v. 3, n. 5 , p. 13-16, 2010.

DAVIES, A. P.; PUFKE, U. S.; ZALUCKI, M. P. Trichogramma (Hymenoptera: Trichogrammatidae) ecology in a tropical Bt transgenic cotton cropping system: sampling to improve seasonal pest impact estimates in the Ord River irrigation area, Australia. Journal of Economic Entomology, v. 102, n. 3, p. 1018-1031, 2009.

FOERSTER, L. A. Seletividade de inseticidas a predadores e parasitóides. In: PARRA, J. R. P. et al. Controle Biológico no Brasil: parasitóides e predadores. São Paulo: Manole, 2002. v. 1, p. 95-114.

GALlO, D. et al. Entomologia agrícola. Piracicaba: FEALQ, 2002. 920 p.

GONÇALVES-GERVÁSIO, R. C. R.; VENDRAMIM, J. D. Efeito de extratos de meliáceas sobre o parasitóide de ovos Trichogramma pretiosum Riley (Hymenoptera: Trichogrammatidae). Neotropical Entomology, v. 33, n. 5, p. 607-612, 2004.

GOULART, R. M. et al. Avaliação da seletividade de inseticidas a Trichogramma spp. (Hymenoptera: Trichogrammatidae) em diferentes hospedeiros. Arquivos do Instituto Biológico, v. 75, n. 1, p. 69-77, 2008.

GRUTZMACHER, A. D. et al. Toxicidade do inseticida altacor sobre Trichogramma pretiosum (Hymenoptera: Trichogrammatidae) em arroz irrigado. In: CONGRESSO BRASILEIRO DE ARROZ IRRIGADO, 7., 2011, Balneário Camboriú. Anais... Itajaí: EPAGRI, 2011. p. 655-658.

INSTITUTOBRASILEIRODEGEOGRAFIAEESTATÍSTICA. Levantamento sistemático da produção agrícola: pesquisa mensal de previsão e acompanhamento das safras agrícolas ao anão civil. Levantamento sistemático da produção agrícola, v. 24, n. 7, p. 1-82, 2011. Disponível em: <http://www.ibge.gov. br/home/estatistica/indicadores/ agropecuaria/lspa/lspa_201107. pdf> Acesso em: 28 ago. 2011.

MAIA, J. B. et al. Selectivity of insecticides used in corn crops to adult Trichogramma atopovirilia. Revista Colombiana de Entomologia, v. 36, n. 2, p. 202-206, 2010.

PINTO, A. S. et al. Comparação de técnicas de liberação de Trichogramma galloi Zucchi (Hymenoptera: Trichogrammatidae) para o controle de Diatraea saccharalis (Fabricius) (Lepidoptera: Crambidae). Neotropical Entomology, v. 32, n. 2, p. 311-318, 2003.

PINTO, A. S.; CANO, M. A.; SANTOS, E. M. A broca-dacana, Diatraea saccharalis. Boletim técnico Biocontrol, v. 1, n. 2, p. 15-20, 2006.

POLANCZYK, R. A. et al. Efeito de Beauveria bassiana (Bals.) Vuillemin e Metarhizium anisopliae (Metsch.) Sorokin nos 
parâmetros biológicos de Trichogramma atopovirilia Oatman \& Platner, 1983 (Hymenoptera: Trichogrammatidae). Ciência e Agrotecnologia, v. 34, n. 6, p. 1412-1416, 2010.

POTRICH, M. et al. Ação de fungos entomopatogênicos sobre o parasitismo de Trichogramma pretiosum Riley, 1879 (Hymenoptera: Trichogrammatidae). Revista Brasileira de Agroecologia, v. 4, n. 2, p. 1995-1998, 2009.

PRATISSOLI, D. et al. Seletividade de inseticidas a Trichogramma pretiosum Riley (Hymenoptera: Trichogrammatidae) em diferentes hospedeiros. Boletín de Sanidad Vegetal Plagas, v. 35, n. 3, p. 347-353, 2009.

SANTOS, A. C.; BUENO, A. F.; BUENO, R. C. O. F. Seletividade de defensivos agrícolas aos inimigos naturais. In: PINTO, A. S. et al. (Ed.). Controle biológico de pragas na prática. Piracicaba: CP2, 2006. p. 221-227.

SHI, Z. H. et al. Evaluation of selective toxicity of five pesticides against Plutella xylostella (Lep: Plutellidae) and their side-effects against Cotesia plutellae (Hym: Braconidae) and
Oomyzus sokolowskii (Hym: Eulophidae). Pest Management Science, v. 60, n. 12, p. 1213-1219, 2004.

SILVA, M. et al. Efeito de fungos entomopatogênicos comerciais sobre o parasitismo de Trichogramma pretiosum Riley (Hymenoptera: Trichogrammatidae). In: SEMINÁRIO SISTEMAS DE PRODUÇÃO AGROPECUÁRIA, 4., 2010, Dois Vizinhos. Anais eletrônicos... Dois Vizinhos: UTFPR, 2010. Disponível em: <https://web.dv.utfpr.edu.br:448/seer/index.php/ SSPA/article/viewFile/508/237>. Acesso em: 08 out. 2011.

STEFANELLO JÚNIOR, G. J. et al. Efeito de inseticidas usados na cultura do milho sobre a capacidade de parasitismo de Trichogramma pretiosum Riley, 1879 (Hymenoptera: Trichogrammatidae). Arquivos do Instituto Biológico, v. 75, n. 2, p. 187-194, 2008.

STERK, G. et al. Results of the seventh joint pesticide testing programme carried out by the IOBC/WPRS-Working Group 'Pesticides and Beneficial Organisms'. BioControl, v. 44, n. 1, p. 99-117, 1999. 